We appreciate the response to this publication feature and welcome all contributions. Contributions may be sent to our Technical Editor Phil Oshel, oshel1pe@cmich.edu

\section{Quality Controls on SEM Performance: A Novel Reference Sample}

\author{
Brendan J. Griffin and Sharon T. Platten \\ Centre for Microscopy and Microanalysis, The University of \\ Western Australia, Crawley, AUSTRALIA \\ Brendan.Griffin@uwa.edu.au
}

The monitoring of performance of an SEM in a multi-user facility is a difficult task as user feedback is generally not reliable. Regular quality control measurements must be undertaken using standard conditions to maintain research-quality products. The test specimens should also be available to the general user community to allow them to monitor their own skills against the reference images. The ready availability of these specimens also helps to eliminate problems related to user specimen preparation, as users can refer back to them when in doubt with respect to SEM performance.

Certified SEM imaging standards are expensive and cannot be provided routinely, particularly where a number of SEM are present. We have investigated a range of potential materials and preparations in search of a low cost, reliable and robust specimen for use in routine (day-to-day) quality control. The other criteria used have been: range in size of detail, suitable detail for image optimisation, homogeneity, suitable feature density and stability under a range of operating conditions and environments.

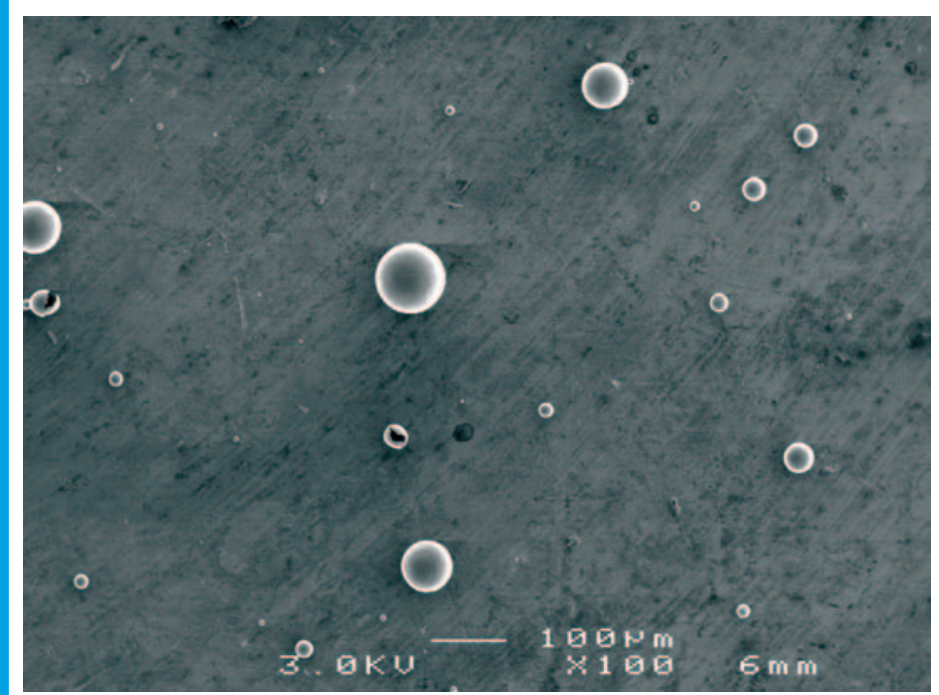

Figure 1: A "Sparky" specimen typical particle distribution resembling GSR. The spheres are usually hollow and occasionally tear, as seen in the small sphere below left of center and another one on the left edge.

Two specimen preparation protocols have been identified as potentially acceptable in this role. "Opal-eyes" is a specimen based on deposits of synthesised 400nm silica spheres decorated with finer gold crystals, on a carbon tab background, which has been reported on earlier (Griffin, et al., 2000). "Sparky" is a specimen prepared by collecting the products from ignition of a standard recreational 'sparkler'. The initial motive for making "sparky" specimens was to

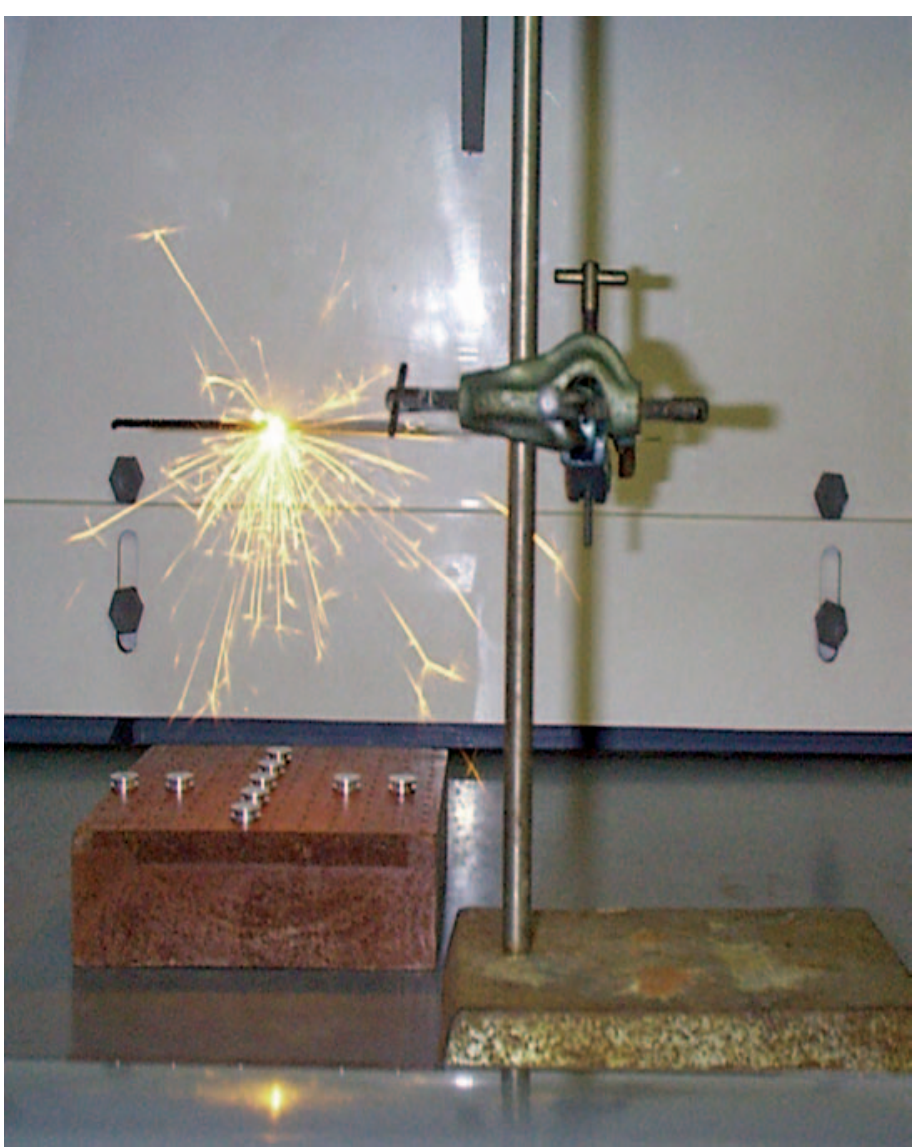

Figure 2: Apparatus for preparing "Sparky" specimens.

prepare stand-in (non-certified) gun-shot residue (GSR) specimens for operator training. The projected particles are fusion products and quench in flight to typically spherical and commonly hollow forms. A range of sizes is present, from around $50 \mu \mathrm{m}$ down to sub-micron (fig 1). Quench structures are often visible and provide a finer level of detail. X-ray analysis of the spheres indicates that they are iron (above detection limit). The particles were collected on carbon tab and polished aluminium (stub) substrates, with good adhesion and coverage on both (fig 2). The density of particle distribution is a function of exposure time, "substrate-to-sparkler" distance, and variability in sparklers fabricated by different vendors. Surprisingly, in view of their composition, the "Sparky" specimens do not require coating and nor do they degrade with time (within our three year frame of reference).

Our data (Platten and Griffin, 2002) indicate that "Sparky" specimens are suitable for routine quality control. Their ready availability and low cost are seen as positive features in a multi-users system, particularly during 'after-hours' when technical support is not available. Besides, they are fun to make!

\section{Reference}

Griffin, B.J., Nockolds, C.E., Phillips, M.R. and Remond, G. (2000): "New needs for imaging and $\mathrm{x}$-ray microanalysis standards: ESEM, CHIME and low voltage microanalysis." Institute of Physics Conference Series \#165 (Proc. of the $2^{\text {nd }}$ meeting of the International Union of Microbeam Analysis Societies - Kona, Hawaii, USA) p.395-6.

Platten, S.T. and Griffin, B.J. (2002): "Quality controls on SEM performance; A comparison of novel reference samples." Proceedings of the 17th Australian Conference on Electron Microscopy, Adelaide, Australia pp. 95. 